\title{
Hyperreflexia in Guillain-Barré syndrome: relation with acute motor axonal neuropathy and anti-GM1 antibody
}

Satoshi Kuwabara, Kazue Ogawara, Michiaki Koga, Masahiro Mori, Takamichi Hattori, Nobuhiro Yuki

\begin{abstract}
Objectives-To investigate the incidence of hyperreflexia in patients with GuillainBarré syndrome (GBS), and its relation with electrodiagnosis of acute motor axonal neuropathy (AMAN), antiganglioside GM1 antibody, and Campylobacter jejuni infection. It was reported that patients with AMAN in northern China often had hyperreflexia in the recovery phase.

Methods-In 54 consecutive Japanese patients with GBS, sequential findings of tendon reflexes were reviewed. By electrodiagnostic criteria, patients were classified as having AMAN or acute inflammatory demyelinating polyneuropathy (AIDP). Anti-GM1 and anti-C jejuni antibodies were measured by enzyme linked immunosorbent assays.
\end{abstract}

Results-Seven (13\%) patients developed hyperreflexia with the spread of the myotatic reflex to other segments in the early recovery phase, one of whom already had hyperreflexia in the acute progressive phase. Of the seven patients, six had AMAN and all seven had anti-GM1 antibodies, whereas only two had anti-C jejuni antibodies. Hyperreflexia was more often found in patients with AMAN than AIDP $(6 / 23 v 1 / 18, p=0.002)$, and in patients with anti-GM1 antibodies than without them $(7 / 26 v 0 / 28, p=0.01)$. Hyperreflexic patients had milder peak disabilities than patients without hyperreflexia $(p=0.03)$. Increased motor neuron excitability in the hyperreflexic patients was supported by increased soleus H-reflex amplitudes and the appearance of $\mathbf{H}$-reflexes in the small hand or foot muscles.

Conclusions-Hyperreflexia often occurs in patients with GBS especially with AMAN, anti-GM1 antibodies, and milder disease. Increased motor neuron excitability further characterises the subgroup of patients with GBS with AMAN and anti-GM1 antibodies.

(F Neurol Neurosurg Psychiatry 1999;67:180-184)

Keywords: Guillain-Barré syndrome; hyperreflexia; acute motor axonal neuropathy; anti-GM1 antibody; H-reflex

Guillain-Barré syndrome (GBS) is an immune mediated polyneuropathy clinically characterised by acute symmetric muscle weakness and areflexia. ${ }^{1}$ Whereas most GBS is demyelinating neuropathy (acute inflammatory demyelinating polyneuropathy, AIDP) in western countries, ${ }^{23}$ an axonal form of GBS, termed acute motor axonal neuropathy (AMAN), has been recognised in northern China ${ }^{45}$ and in other countries. ${ }^{6}{ }^{7}$ It is suggested that AMAN is associated with pure motor axonal involvement, antiganglioside GM1 antibodies, or preceding Campylobacter jejuni infection. ${ }^{7-9}$

Chinese patients with AMAN are reported to often develop hyperreflexia during the early phase of recovery, ${ }^{4}$ and other studies described patients with acute motor neuropathy with preserved tendon reflexes. ${ }^{10-12}$ Preserved or exaggerated tendon reflexes do not usually occur in patients with AIDP and in patients with other peripheral neuropathies. We, therefore, investigated the incidence of hyperreflexia and its relation with electrophysiological subtypes (AMAN or AIDP), anti-GM1 antibodies, or $C$ jejuni infection in consecutive patients with GBS in Japan.

\section{Patients and methods}

PATIENTS

Fifty four patients with GBS who were seen at Chiba University Hospital and its affiliated hospitals between January 1992 and April 1998 were studied. The mean age was 40.1 years (range 3 to 78 years). Their first neurological examination and electrodiagnostic study were done within 2 weeks of onset. They fulfilled the clinical criteria for GBS, ${ }^{1}$ except for two patients who had normal or brisk tendon reflexes in the first examination. The two patients were diagnosed as having AMAN because of electrophysiological evidence of motor axonal loss. Clinical disability was evaluated with the Hughes functional grading scale ${ }^{13}$ and patients were followed up for 3 months after onset. Hyperreflexia was regarded as present when there were increased amplitudes of tendon reflexes, abnormal reflex spread (finger jerks after tendon tapping of the biceps brachii or brachioradialis muscle, or thigh adduction after patella tendon tapping), and decreased reflex threshold (biceps contraction after tendon tapping of the brachioradialis, or quadriceps contraction after tapping of the midportion of the patella). Hoffmann's and Babinski's signs were also examined.

\section{ELECTROPHYSIOLOGY}

Nerve conduction and $\mathrm{H}$-reflex studies were done sequentially using the conventional procedures. AMAN or AIDP was diagnosed based 
Table 1 Clinical and laboratory features of patients with GBS and hyperreflexia

\begin{tabular}{llllllll}
\hline & & & & & & & \multicolumn{2}{l}{\begin{tabular}{l} 
Antibody titre to ganglioside GM1 \\
\cline { 6 - 7 } Patient No
\end{tabular}} & Age/sex & $\begin{array}{l}\text { Prodromal } \\
\text { symptom }\end{array}$ & $\begin{array}{l}\text { Cranial } \\
\text { nerve palsy }\end{array}$ & \multicolumn{2}{l}{$\begin{array}{l}\text { Sensory } \\
\text { loss }\end{array}$} & Electrodiagnosis & IgG & IgM & Antitre to \\
Campylobacter jejuni
\end{tabular}

$\mathrm{AMAN}=$ acute motor axonal neuropathy; $\mathrm{AIDP}=$ acute inflammatory demyelinating polyneuropathy; Blank=negative

on the electrodiagnostic criteria described by Ho et al. ${ }^{14}$ When, besides the AMAN pattern, amplitude reduction of sensory nerve action potentials was present, the patients were classified as having acute motor-sensory axonal neuropathy (AMSAN). When nerve conduction studies were done more than once within 2 weeks of onset, the results of the last study were used for the electrodiagnostic classification.

The H-reflex in the soleus muscle was recorded after submaximum stimulation delivered to the tibial nerve at the knee. ${ }^{15}$ Stimulus duration was $1.0 \mathrm{~ms}$ and the rate was $0.5 \mathrm{~Hz}$. Patients lay in the prone position with the ankle joint maintained at approximately $120^{\circ}$. The ratio of the peak to peak maximum $\mathrm{H}$-reflex to the maximum compound muscle action potential $(\mathrm{H} / \mathrm{M}$ ratio) was measured. Whether the $\mathrm{H}$-reflex was elicited in the abductor pollicis brevis or abductor hallucis muscle, was also examined after stimulation at the wrist or ankle respectively. The $\mathrm{H} / \mathrm{M}$ ratio control data were obtained from 15 normal volunteers aged 21 to 52 years (mean 38.6 years).

ANTI-GM1 ANTIBODY ASSAY

Serum samples obtained in the first 4 weeks after onset were frozen at $-80^{\circ} \mathrm{C}$ until used. The serum samples were tested for the presence of $\operatorname{IgG}$ and $\operatorname{IgM}$ antibodies to GM1 by the enzyme linked immunosorbent assay as described elsewhere. ${ }^{16}$ Well characterised human serum with high IgG anti-GM1 antibody titre was the internal control. Antibody titre was the highest serum dilution at which the optical density at $492 \mathrm{~nm}$ was 0.1 or more. Serum was considered positive when the titre was $1: 500$ or more.

ANTI-CAMPYLOBACTER JEJUNI ANTIBODY ASSAY IgG anti- $C$ jejuni antibody was measured by the enzyme linked immunosorbent assay as described elsewhere. ${ }^{17}$ Serum was considered

Table 2 Relation between hyperreflexia and electrodiagnosis or Anti-GM1 positivity

\begin{tabular}{llll}
\hline & \multicolumn{2}{c}{ Hyperreflexia } & \\
\cline { 2 - 3 } & Present $(n=7)$ & Absent $(n=47)$ & $p$ Value \\
\hline AMAN & 6 & 17 & 0.002 \\
AMSAN & 0 & 2 & 0.18 \\
AIDP & 1 & 17 & \\
Unclassified & 0 & 11 & 0.01 \\
Anti-GM1: & 7 & 21 & \\
$\quad$ Positive & 0 & 26 & \\
\hline
\end{tabular}

AMAN=acute motor axonal neuropathy; AMSAN=acute motor and sensory neuropathy; $\mathrm{AIDP}=$ acute inflammatory demyelinating polyneuropathy. positive when the titre was $1: 2000$ or more. The IgG anti- $C$ jejuni antibody assay is sufficiently sensitive and specific for serological evidence of $C$ jejuni infection, even if IgM and IgA antibodies are not determined. ${ }^{17}$ All anti-GM1 and anti-C jejuni antibody assays were done by one investigator (MK) who was blinded to the clinical and electrophysiological data.

STATISTICAL ANALYSIS

Differences in percentages were tested with $\chi^{2}$ or Fisher's exact test, and in medians with the Mann-Whitney $U$ test using Stat View 4.5 software.

\section{Results}

TENDON REFLEX AND H-REFLEX

In the first examination ( 3 to 14 days after onset, mean 7.9 days), 52 of the 54 patients had areflexia $(n=44)$ or hyporeflexia $(n=8)$. Of the remaining two patients, one had normal reflexes, and the other, hyperreflexia (patient 6 in tables 1 and 2). During weeks 3 to 4 , seven patients $(13 \%)$, including patient 6 , developed
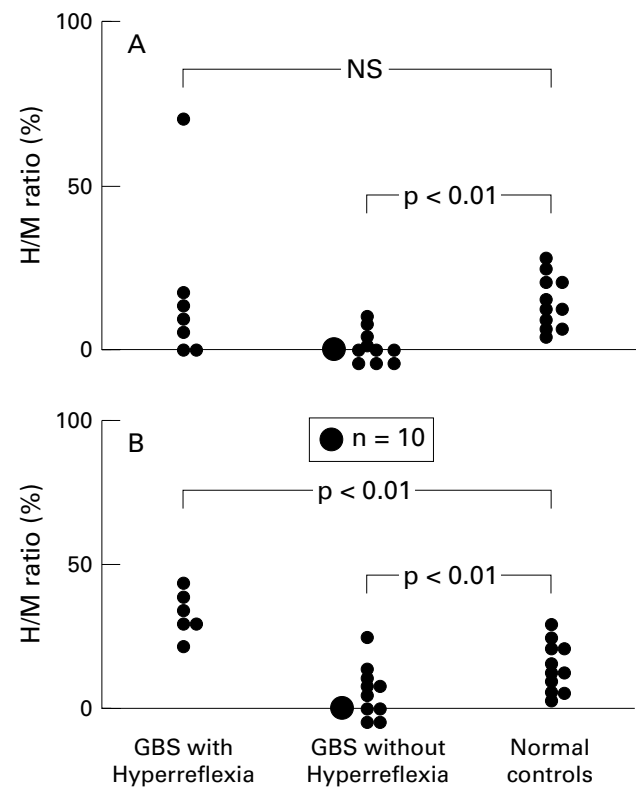

Figure 1 Ratio of the maximum $H$ reflex to the maximum compound muscle action potential (H/M ratio) in patients with $G B S$ and normal subjects. In the acute phase $(A)$, the ratios did not differ significantly between patients with GBS who later developed hyperreflexia and normal subjects, whereas patients with GBS without hyperreflexia had significantly low ratios. In the recovery phase (B), the ratios were significantly increased in the patients with $G B S$ with hyperreflexia $(p<0.01)$ but decreased in the other patients with GBS without hyperreflexia $(p<0.01)$, compared with those of the normal subjects. 

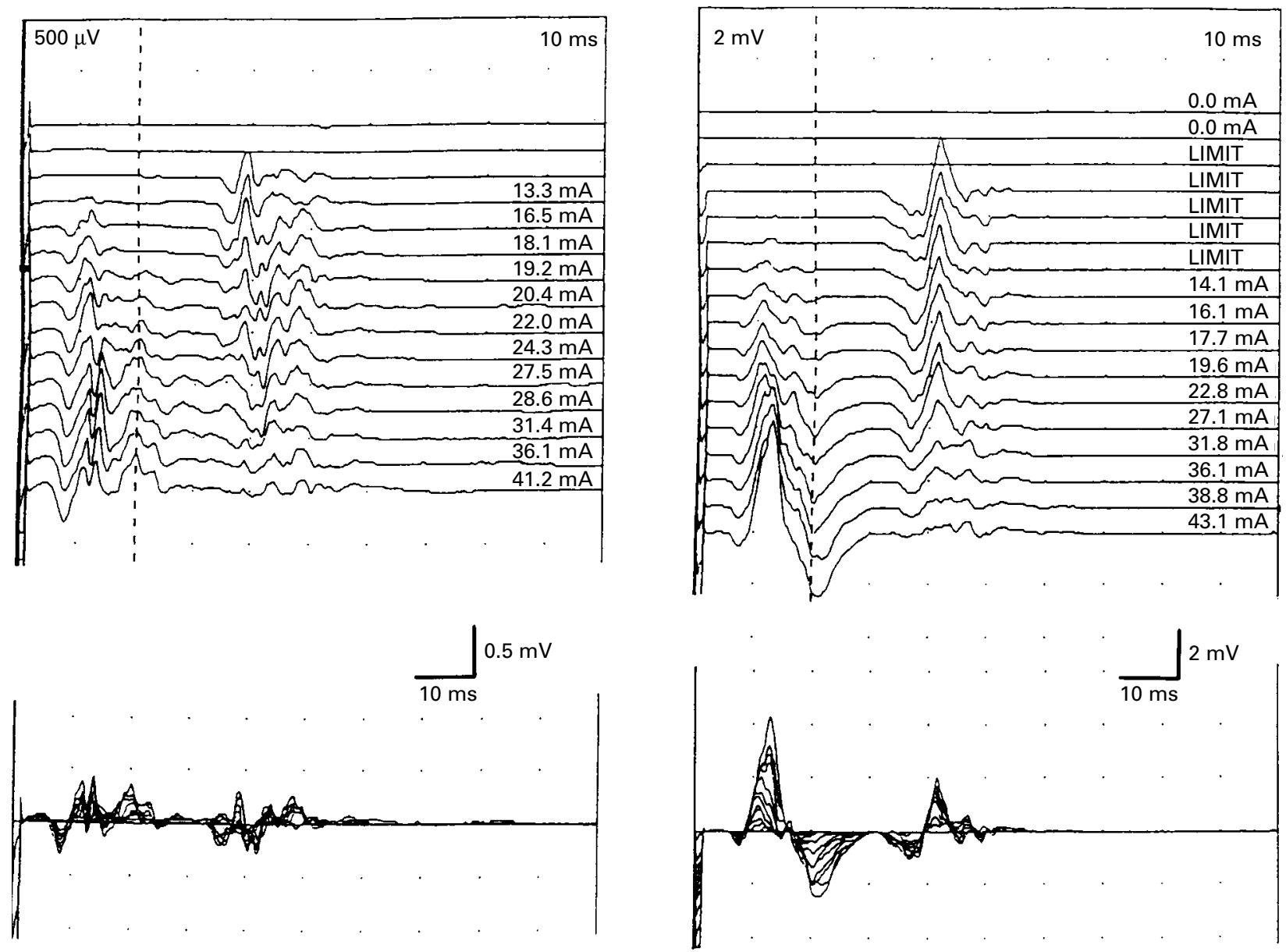

Figure 2 Soleus $H$-reflexes in patient 4 recorded on day 8 (left) when achilles tendon reflexes were absent and on day 40 (right) when the reflexes were hyperactive. Left traces: peak to peak amplitude of compound muscle action potential $(C M A P)=0.742 \mathrm{mV}$; H-reflex amplitude $=0.571 \mathrm{mV}$; H/M ratio $=0.77$. Right traces: $C M A P$ amplitude $=1.724 \mathrm{mV} ; \mathrm{H}$ reflex amplitude $=0.785 \mathrm{mV} ; \mathrm{H} / \mathrm{M}$ ratio $=0.46$. Note the increased $\mathrm{H} / \mathrm{M}$ ratio despite the marked reduction in the CMAP in the left traces (normal mean (SD) of H/M ratio, 0.17 (SD 0.08)). Lowest traces: superimposed responses.

hyperreflexia (table 1). Hyperreflexia was associated with abnormal reflex spread and decreased reflex threshold. Hoffmann's sign were present in four patients, but none had Babinski’s sign.

Figure 1 shows the soleus $\mathrm{H} / \mathrm{M}$ ratios of patients with GBS in the acute (weeks 1 to 3) and recovery (weeks 5 to 8 ) phases, and those of the normal controls. In the acute phase, $\mathrm{H}$ reflexes were recordable in only 10 patients, including six who developed hyperreflexia. patients with GBS had low $\mathrm{H} / \mathrm{M}$ ratios, except for patient 4, who had a high ratio of 0.77 (fig 2). In the recovery phase, the $H / M$ ratios were significantly higher in patients with GBS with hyperreflexia and significantly lower in the other patients with GBS, compared with the ratios of the normal subjects. Of the seven patients with hyperreflexia, H-reflexes were elicited in the abductor pollicis brevis in four and in the abductor hallucis in three (fig 3).

ELECTRODIAGNOSIS

Patients were classified as having AMAN $(n=23)$, AIDP $(n=18)$, or AMSAN $(n=2)$, or were unclassified $(n=11)$. Of the seven patients with hyperreflexia, six had AMAN and one AIDP (table 1). Sequential findings of patella tendon reflexes in AMAN and patients with AIDP are shown in fig 4 . In the acute phase (week 1 or 2 ), $65 \%$ of AMAN, and $100 \%$ of patients with AIDP showed absence of patella tendon reflexes. During the early recovery phase (weeks 4 to 6 ), $34 \%$ of patients with AMAN and $12 \%$ of patients with AIDP had normal or brisk patella tendon reflexes. Most patients $(88 \%)$ with AIDP still had areflexia 3 months after the onset. Two patients with AMSAN had areflexia up to 3 months after the onset. Hyperreflexia was significantly more frequent in AMAN (table 2).

\section{ANTI-GM1 ANTIBODY}

Positive anti-GM1 antibodies were found in 28 (52\%) patients; 21 had IgG alone, four IgM alone, and three had both classes. All the seven patients with hyperreflexia had anti-GM1 antibodies, most of which were of IgG class (table 1). Hyperreflexia was significantly more frequent in patients with than without anti-GM1 antibodies (table 2).

ANTI-C FEFUNI ANTIBODY

Positive anti- $C$ jejuni antibodies were found in $13(24 \%)$ patients. Hyperreflexia was present in two $(15 \%)$ of the 13 patients with anti- $C$ jejuni antibodies, and in five (14\%) of 36 


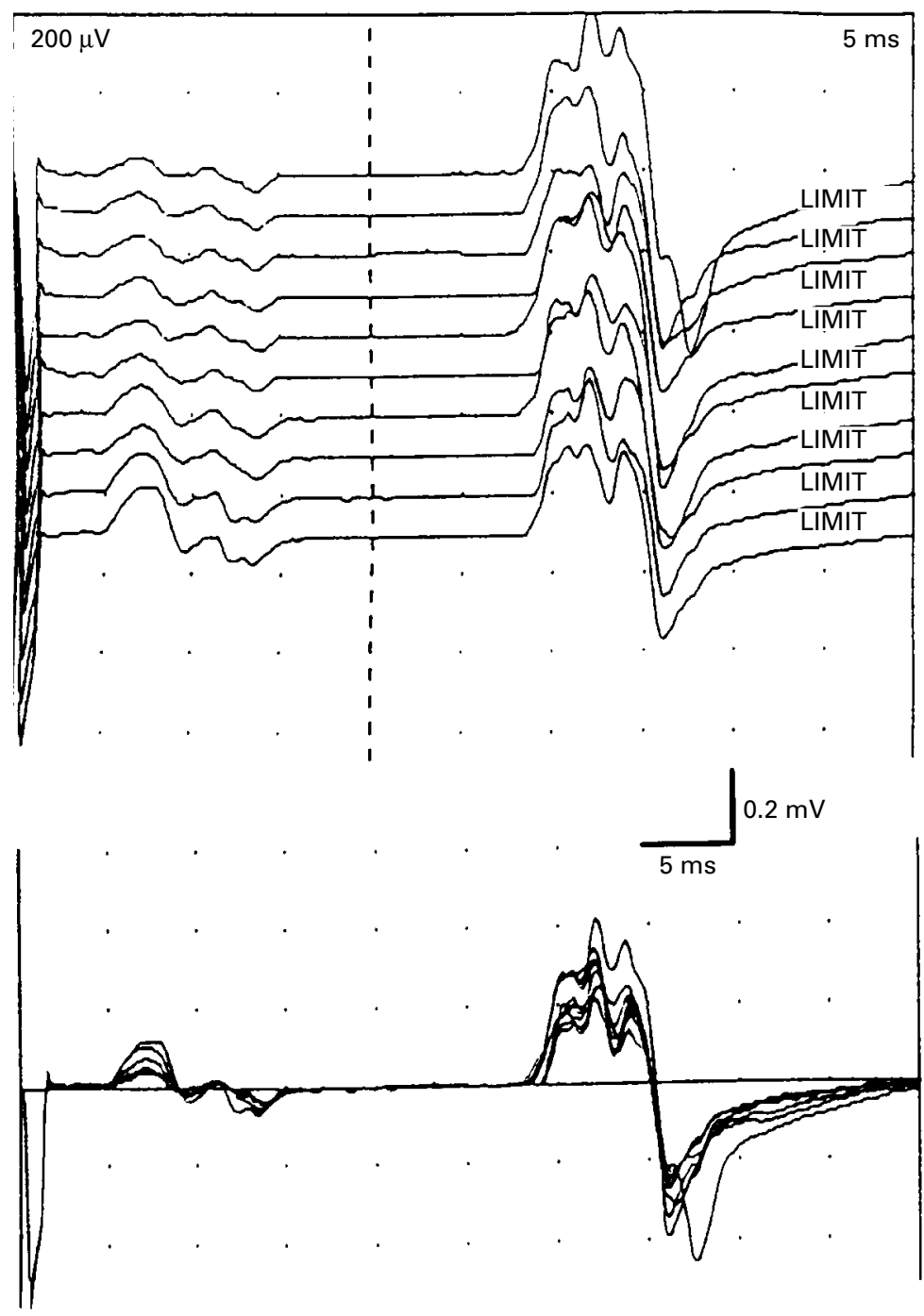

Figure 3 H-reflexes in the abductor pollicis brevis muscle of patient 3 (day 55). Lowest traces: superimposed responses. patients without anti- $C$ jejuni antibody. $C$ jejuni infection, therefore, had no relevant association with hyperreflexia.

\section{CLINICAL FEATURES OF PATIENTS WITH}

HYPERREFLEXIA

The clinical manifestations of the patients with hyperreflexia were similar to those of the other patients with AMAN; less frequent cranial and sensory nerve involvement (table 1). They had, however, significantly less peak disabilities than the other patients with GBS. On the Hughes functional grading scale, the median (range) in patients with hyperreflexia was 2.5 (2.0-3.0), whereas it was $3.5(2.0-5.0)$ in patients without hyperreflexia $(\mathrm{p}=0.03)$. Of the hyperreflexic patients, three were able to walk with aids (Hughes grade 3), and the other four were able to walk independently (Hughes grade 2 ). The three patients were treated with intravenous immunoglobulin infusion (2 and 4 ) or plasmapheresis (1). All seven patients recovered well, six being able to run 3 months after onset. Two patients ( 3 and 5 ) had moderate atrophy of the small hand muscles 3 months after onset.

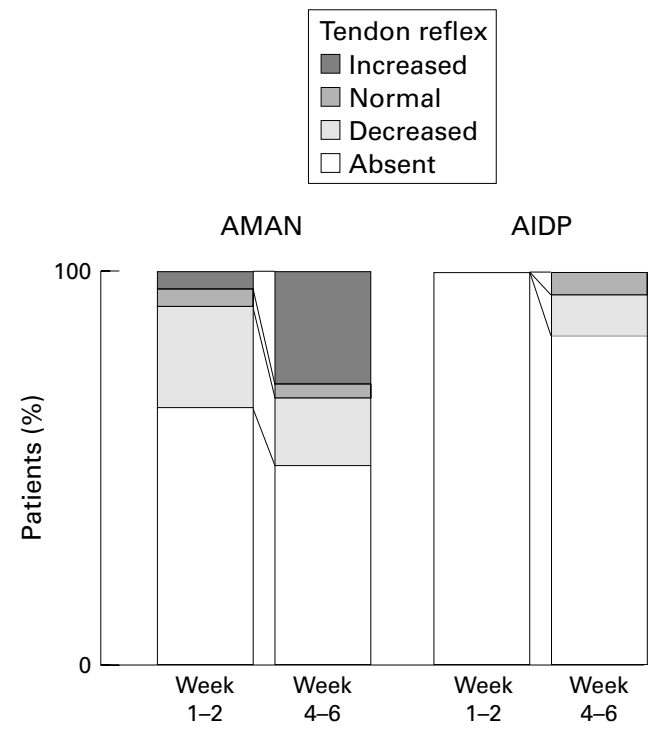

Figure 4 Sequential findings of patella tendon reflexes in patients with acute motor axonal neuropathy ( $A M A N)$ or acute inflammatory demyelinating polyneuropathy (AIDP).

\section{Discussion}

Our study showed that some patients with GBS showed obvious hyperreflexia when they had the AMAN pattern, anti-GM1 antibody, and milder muscle weakness. The incidence of hyperreflexia in AMAN was 33\%, whereas McKahnn et al. ${ }^{5}$ found that $12(48 \%)$ of 25 Chinese patients with AMAN showed hyperreflexia. Most of the Chinese patients were children, but our findings showed a similar incidence of hyperreflexia in AMAN that occurs in adults.

Because sensory nerve involvement in AIDP or AMAN should contribute to the areflexia, the selective involvement of motor axons in AMAN could account for the relative preservation or earlier appearance of reflexes ${ }^{18}$; but it does not account for hyperreflexia and reflex spread to other segments. Some of our patients with AMAN had electrophysiological evidence of increased motor neuron excitability shown by the increased soleus $\mathrm{H} / \mathrm{M}$ ratios and the abnormal appearance of $\mathrm{H}$-reflexes in the small muscles of the hands or feet. Our hyperreflexic patients had less peak disability than the other patients with GBS. We consider that hyperreflexia could be evident when motor axonal loss is less severe and sensory nerves are intact. Van der Meché et $a l^{19}$ found that, in patients with pure motor GBS, tendon reflexes were preserved up to MRC grade 3 paresis.

The mechanism that causes hyperreflexia in GBS is unknown. The abnormal reflex spread to other segments suggests a central mechanism; possibly due to the dysfunction of spinal inhibitory interneurons, or of the upper motor neurons. Because hyperreflexia was found only in patients with anti-GM1 antibodies, we speculate a possible role of this antibody. Hyperreflexia is occasionally seen in chronic motor neuropathy associated with high titre anti-GM1 antibody, ${ }^{20}{ }^{21}$ and anti-GM1 serum injected in the subarachnoid space was reported to cause damage to the central axons in 
the spinal cord as well as nerve roots. ${ }^{22}$ In addition, the spinal nerve roots, where the bloodnerve barrier is anatomically deficient, ${ }^{23}$ are preferentially affected in GBS. ${ }^{24}$ Inflammation in the spinal roots may lead to local dysfunction of the blood-CNS barrier and allow anti-GM1 antibodies to bind with the neural structures in the spinal cord. Further study is required to clarify the mechanism of increase in motor neuron excitability in GBS.

In AMAN or anti-GM1 positive GBS, excitability of the motor neuron could increase even in the acute progressive phase. In most of our patients, hyperreflexia developed in the early recovery phase, but a few patients with AMAN had brisk reflexes in the acute phase. Moreover, patient 4 had an increased soleus $\mathrm{H} / \mathrm{M}$ ratio despite the markedly low compound muscle action potential at the time when achilles tendon reflexes were absent (fig 2, on day 8), suggestive of hyperexcitability of the residual motor neurons. Several studies described patients who had acute pure motor neuropathy with preserved tendon reflexes throughout the course, ${ }^{10-12}$ and in some cases, the disorder was not diagnosed as GBS because of preserved reflexes. ${ }^{11}$ Yuki and Hirata ${ }^{12}$ described four patients with AMAN with preserved tendon reflexes (two of them had even brisk reflexes), and proposed that these patients should be treated as having GBS. Our results confirm that hyperreflexia is not a finding inconsistent with the diagnosis of GBS.

In conclusion, hyperreflexia often occurs in patients with GBS with AMAN, anti-GM1 antibody, and less severe disease. Increased motor neuron excitability further characterises the subgroup of patients with GBS with the AMAN pattern and anti-GM1 antibodies.

1 Asbury AK, Cornblath DR. Assessment of current diagnostic criteria for Guillain-Barré syndrome. Ann Neurol 1990;27(suppl):S21-4.

2 Vriesendorp FJ, Mishu B, Blaser MJ, et al. Serum antibodies to GM1, GDlb, peripheral myelin, Campylobacter jejuni and in patients with Guillan-Barré syndrome and controls: correlation and prognosis. Ann Neurol 1993;34: 130-5.

3 Rees JH, Gregson NA, Hughes RAC. Anti-GM1 antibodies in Guillain-Barré syndrome and their relationship to Campylobacter jejuni infection. Ann Neurol 1995;38:80916.
4 McKhann GM, Cornblath DR, Ho TW, et al. Clinical and electrophysiological aspects of acute paralytic disease of children and young adults in northern China. Lancet 1991; 338:593-7.

5 McKahnn GM, Cornblath DR, Griffin JW, et al. Acute motor axonal neuropathy: a frequent cause of acute flaccid paralysis in China. Ann Neurol 1993;33:333-42.

6 Visser LH, van der Meché FGA, van Doorn PA, et al. Guillan-Barré syndrome without sensory loss (acute motor neuropathy). A subgroup with specific clinical, electrodiagnostic and laboratory features. Brain 1995;118:841-7.

7 Kuwabara S, Yuki N, Koga M, et al. IgG anti-GM1 antibody is associated with reversible conduction failure and axonal degeneration in Guillan-Barré syndrome. Ann Neurol 1998;44:202-8

8 Kornberg AJ, Pestronk A, Bieser K, et al. The clinical correlates of high-titer IgG anti-GM1 antibodies. Ann Neurol 1994;35:234-7.

9 Jacobs BC, van Doorn PA, Schmitz PIM, et al. Campylobacter jejuni infections and anti-GM1 antibodies in Guillain-Barré syndrome. Ann Neurol 1996;40:181-7.

10 Pryor WM, Freiman JS. Guillain-Barré syndrome associated with Campylobacter infection. Aust $N Z$ F Med 1984; 14:687-8.

11 Jackson CE, Barohn RJ, Mendell JR. Acute paralytic syndrome in three American men. Comparison with syndrome in three American men. Con
Chinese cases. Arch Neurol 1993;50:732-5.

12 Yuki N, Hirata K. Preserved tendon reflexes in Campylobacter neuropathy [letter]. Ann Neurol 1998;43:546-7.

13 Guillain-Barré syndrome steroid trial group. Double blind trial of intravevous methylprednisolone in Guillan-Barré syndrome. Lancet 1993;341:586-90.

14 Ho TW, Mishu B, Li CY, et al. Guillain-Barré syndrome in northern China. Relationship to Campylobacter jejuni infection and anti-glycolipid antibodies. Brain 1995;118: 597-605.

15 Fisher MA. AAEM minimonograph No 13: $\mathrm{H}$ reflexes and F waves: physiological and clinical indications. Muscle Nerve 1992;15:1223-33.

16 Yuki N. Acute paresis of extraocular muscles associated with IgG anti-GQ1b antibody. Ann Neurol 1996;39:668-72.

17 Koga M, Yuki N, Takahashi M, et al. Close association of IgA anti-ganglioside GM1 antibodies with antecedent Campylobacter jejuni infection in Guillain-Barré and Fisher's syndromes. F Neuroimmunol 1998;81:138-43.

18 Asbury AK, Cornblath DR. Preserved tendon reflexes in Campylobacter neuropathy [reply to letter]. Ann Neurol 1998;43:547.

19 van der Meché FGA, Meulstee J, Vermeulen M, et al. Patterns of conduction failure in the Guillain-Barré syndrome. Brain 1988;111:405-16.

20 Pestronk A, Cornblath DR, Ilyas AA, et al. A treatable multifocal motor neuropathy with antibodies to GM1 ganglioside. Ann Neurol 1988;24:73-8.

21 Kinsella LJ, Lange DJ, Trojaborg W, et al. Clinical and electrophysiologic correlates of elevated anti-GM1 antibody titers. Neurology 1994;44:1278-82.

22 Schwerer B, Lassmann H, Kitz K, et al. Ganglioside GM1, a molecular target for immunological and toxic attacks: similarity of neuropathological lesions induced by gangliosideantiserum and cholera toxin. Acta Neuropathol 1986;72:5561

23 Olsson Y. Topographical difference in the vascular permeability of the peripheral nervous system. Acta Neuropathol 1968;10:26-33.

24 Brown WF, Snow R. Patterns and severity of conduction abnormalities in Guillain-Barré syndrome. $\mathcal{F}$ Neurol Neurosurg Psychiatry 1991;54:768-74. 